APJL IN PRESS

Preprint typeset using LTEX style emulateapj v. 08/22/09

\title{
TWO-DIMENSIONAL FULL PARTICLE SIMULATION OF A PERPENDICULAR COLLISIONLESS SHOCK WITH A SHOCK-REST-FRAME MODEL
}

\author{
TAKAYUKI UMEDA ${ }^{1}$, MASAHIRO YAMAO ${ }^{1}$, AND RYO YAMAZAKI ${ }^{2}$ \\ ApJL in press
}

\begin{abstract}
A two-dimensional (2D) shock-rest-frame model for particle simulations is developed. Then full kinetic dynamics of a perpendicular collisionless shock is examined by means of a 2D full particle simulation. We found that in the 2D simulation there are fewer nonthermal electrons due to surfing acceleration which was seen in the previous 1D simulations of a high Mach number perpendicular shock in a low-beta and weakly magnetized plasma. This is because the particle motion along the ambient magnetic field disturbs the formation of coherent electrostatic solitary structures which is necessary for electron surfing acceleration.
\end{abstract}

Subject headings: acceleration of particles — methods: numerical — shock waves — supernova remnants

\section{INTRODUCTION}

Observations of shell-type supernova remnants (SNRs) provide us evidence for electron acceleration at SNR shocks, and in some cases the maximum energy of the electrons exceeds $\sim 10 \mathrm{TeV}$ (Koyama et al. 1995; Bamba et al.2003, 2005). The most plausible mechanism of the acceleration is the diffusive shock acceleration (DSA), which explains the broadband power-law spectrum with an index around 2 (Drury 1983; Blandford \& Eichler 1987). Before the DSA phase in which electrons cross the shock front many times, they have to be pre-accelerated by an unknown injection mechanism. This "injection problem" is still unresolved.

One of the possible injection mechanisms is the shock surfing acceleration (Shimada \& Hoshino 2000; Hoshino \& Shimada 2002). Shimada \& Hoshino (2000) performed a one-dimensional (1D) electromagnetic full particle simulation and found the formation of large-amplitude electrostatic solitary structures during the cyclic reformation of a high Mach number perpendicular shock in a low-beta and weakly magnetized plasma. The coherent solitary structures trap electrons in their electrostatic potential well, resulting in significant surfing acceleration of electrons. The electron surfing has been regarded as an efficient direct acceleration mechanism at SNR shocks and studied by many authors (e.g., McClements et al. 2001; Hoshino \& Shimada 2002; Schmitz et al. 2002a b; Amano \& Hoshino 2007). However, these previous works are based on 1D simulations, in which configuration of shock magnetic fields cannot be modified. Since charged particles can easily drift along magnetic fields, it is important to take into account the modification of shock magnetic fields and the free motion of particles along shock magnetic fields. Hence, multi-dimensional simulations are necessary.

In the present study we perform a $2 \mathrm{D}$ full particle simulation of a high Mach number and low-beta perpendicular collisionless shock. The preliminary result shows that the electron surfing acceleration is suppressed in a 2D system.

\section{FULL PARTICLE SIMULATION}

\footnotetext{
${ }^{1}$ Solar-Terrestrial Environment Laboratory, Nagoya University, Nagoya, 464-8601, Japan; umeda@ stelab.nagoya-u.ac.jp

${ }^{2}$ Department of Physical Science, Hiroshima University, HigashiHiroshima 739-8526, Japan; ryo@ theo.phys.sci.hiroshima-u.ac.jp
}

Generally speaking, it is difficult to perform multidimensional simulations of collisionless shocks even with present-day supercomputers. In the previous simulations of high Mach number shocks (e.g., Shimada \& Hoshino 2000; Schmitz et al. 2002a b; Lee et al. 2004; Amano \& Hoshino 2007), a collisionless shock is excited with the "injection method" (also called the reflection, wall, or piston method), in which a plasma is injected from one side of the simulation domain and is reflected back when it reaches the other side. A collisionless shock is excited by interaction between injected and reflected plasmas. Thus, the simulation domain is taken in the downstream rest frame, and the excited shock wave propagates upstream. It is necessary to take a very long simulation domain in the propagation direction of the shock wave in order to study long-time evolution of moving shock waves.

Recently, 1D full particle simulations have been performed in an almost shock rest frame (Muschietti \& Lembege 2006; Umeda \& Yamazaki 2006). In these simulations, a collisionless shock is excited with the "relaxation method," which was first used in hybrid code simulations (Leroy et al. 1981, 1982). Muschietti \& Lembege (2006) used the Darwin approximation in which the displacement current due to the transverse electric field is neglected, while Umeda \& Yamazaki (2006) solved the full set of Maxwell's equations to take into account all the plasma waves. Although the shock-rest-frame model is useful to perform simulations of shocks with less computer resources, there have been few multi-dimensional shock-rest-frame models. Here, we successfully extended the previous 1D shock-rest-frame model (Umeda \& Yamazaki 2006) to two dimensions.

We use a 2D electromagnetic particle code (Umeda 2004), in which the full set of Maxwell's equations and the relativistic equation of motion for individual electrons and ions are solved in a self-consistent manner. The continuity equation for charge is also solved to compute the exact current density given by the motion of charged particles (Umeda et al. 2003).

The initial state consists of two uniform regions separated by a discontinuity. In the upstream region that is taken in the left-hand side of the simulation domain, electrons and ions are distributed uniformly in space and are given random velocities $\left(v_{x}, v_{y}, v_{z}\right)$ to approximate shifted Maxwellian momentum distributions with the drift velocity $u_{x 1}$, number density $n_{1} \equiv \epsilon_{0} m_{e} \omega_{p e 1}^{2} / e^{2}$, and isotropic temperatures $T_{e 1} \equiv m_{e} v_{t e 1}^{2}$ 
and $T_{i 1} \equiv m_{i} v_{t i 1}^{2}$, where $m, e, \omega_{p}$, and $v_{t}$ are the mass, charge, plasma frequency, and thermal velocity, respectively. Subscripts " 1 " and " 2 " denote "upstream" and "downstream," respectively. The upstream magnetic field $B_{y 01} \equiv-m_{e} \omega_{c e 1} / e$ is also assumed to be uniform, where $\omega_{c}$ is the cyclotron frequency (with sign included). The downstream region taken in the right-hand side of the simulation domain is prepared similarly with the drift velocity $u_{x 2}$, density $n_{2}$, isotropic temperatures $T_{e 2}$ and $T_{i 2}$, and magnetic field $B_{y 02}$.

We take the simulation domain in the $x-y$ plane and assume a perpendicular shock (i.e., $B_{x 0}=0$ ). Since the ambient magnetic field is taken in the $y$-direction, free motion of particles along the ambient magnetic field is taken into account. As a motional electric field, a uniform external electric field $E_{z 0}=-u_{x 1} B_{y 01}=-u_{x 2} B_{y 02}$ is applied in both upstream and downstream regions, so that both electrons and ions drift in the $x$-direction. At the left boundary of the simulation domain in the $x$-direction, we inject plasmas with the same quantities as those in the upstream region, while plasmas with the same quantities as those in the downstream region are also injected from the right boundary in the $x$-direction. We adopted absorbing boundaries to suppress nonphysical reflection of electromagnetic waves at both ends of the simulation domain in the $x$-direction (Umeda et al.2001), while the periodic boundaries are imposed in the $y$-direction.

The initial downstream quantities are given by solving the shock jump conditions for a magnetized two-fluid isotropic plasma consisting of electrons and ions (e.g., Hudson 1970). In order to determine a unique initial downstream state, we need given upstream quantities $u_{x 1}, \omega_{p e 1}, \omega_{c e 1}, v_{t e 1}$, and $T_{i 1} / T_{e 1}$ and another parameter. We assume a low-beta and weakly magnetized plasma such that $\beta_{e 1}=\beta_{i 1}=0.125$ and $\omega_{c e 1} / \omega_{p e 1}=-0.05$ in the upstream region. We also use a reduced ion-to-electron mass ratio $m_{i} / m_{e}=25$ for computational efficiency. The light speed $c / v_{t e 1}=80.0$ and the bulk flow velocity of the upstream plasma $u_{x 1} / v_{t e 1}=8.0$ are also assumed. Then, the Alfvén Mach number is calculated as $M_{A}=\left(u_{x 1} / c\right)\left|\omega_{p e 1} / \omega_{c e 1}\right| \sqrt{m_{i} / m_{e}}=10.0$. The ion-toelectron temperature ratio in the upstream region is given as $T_{i 1} / T_{e 1}=1.0$. Note that these parameters are almost the same as the previous full particle simulations of a perpendicular shock (Shimada \& Hoshino 2000; Hoshino \& Shimada 2002; Schmitz et al. 2002a.b; Lee et al. 2004). In this study, the downstream ion-to-electron temperature ratio $T_{i 2} / T_{e 2}=8.0$ is also assumed as an initial parameter to obtain the unique downstream quantities $\omega_{p e 2} / \omega_{p e 1}=1.953, \omega_{c e 2} / \omega_{p e 1}=0.191$, $u_{x 2} / v_{t e 1}=2.097$, and $v_{t e 2} / v_{t e 1}=5.715$.

We used $N_{x} \times N_{y}=4096 \times 64$ cells for the upstream region and $N_{x} \times N_{y}=2048 \times 64$ cells for the downstream region. The grid spacing and time step of the present simulation are $\Delta x / \lambda_{e 1}=1.0$ and $\omega_{p e 1} \Delta t=0.005$. We used 16 pairs of electrons and ions per cell in the upstream region and 64 pairs of electrons and ions per cell in the downstream region at the initial state. One might think that the downstream system size is too short because the downstream does have an affect on the transition region. However, we have confirmed by changing the downstream system size that there is not any critical change in the simulation result when the downstream system size is longer than the typical ion gyroradius $u_{x 1} / \omega_{c i 2}$ (Umeda \& Yamazaki 2006). This implies that the results are most appropriate to the transition region, and that the transition region, is approximately modeled by the present shockrest-frame model despite the lack of an extensive downstream

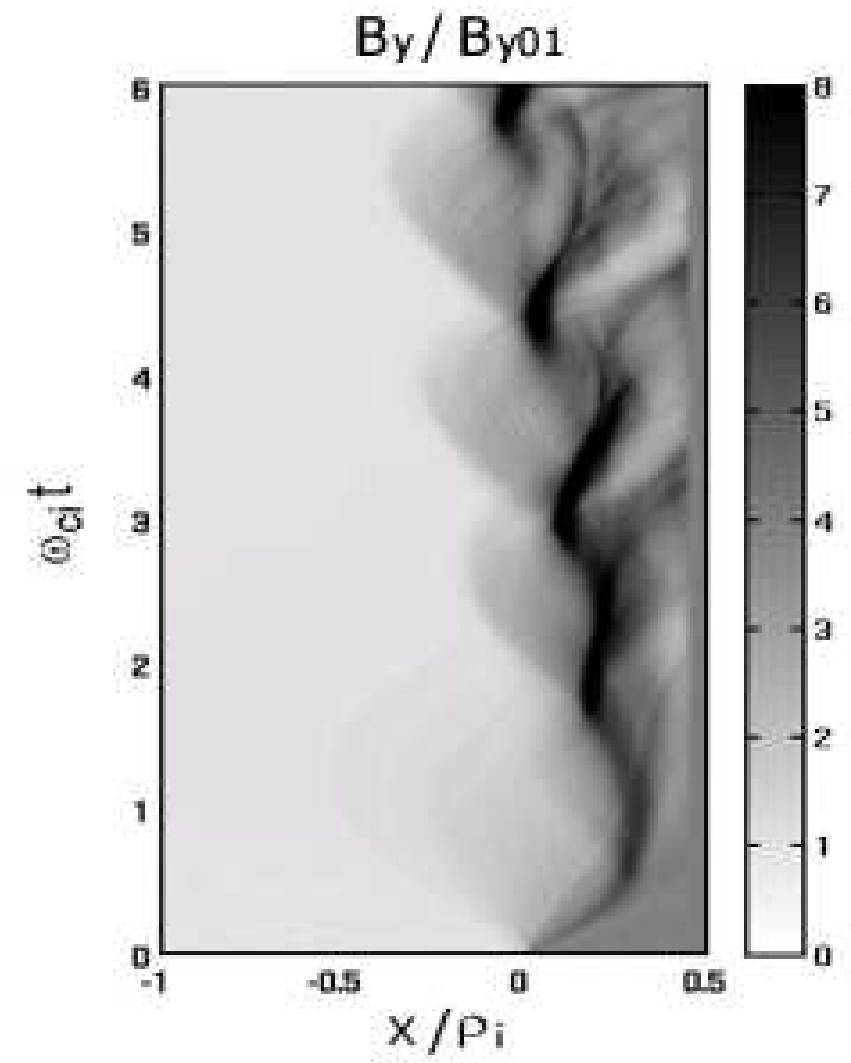

FIG. 1.- Transverse magnetic field $B_{y}$ as a function of position $x$ and time $t$ on the $x$-axis $(y=0)$. The position and time are normalized by $\rho_{i} \equiv u_{x 1} / \omega_{c i 1}$ and $1 / \omega_{c i 1}$, respectively. The magnitude is normalized by the initial upstream magnetic field $B_{y 01}$.

region. Thus, the processes seen in the present simulation should be robust.

\section{RESULT}

Figure 1 shows the transverse magnetic field $B_{y}$ as a function of position $x$ and time $t$ on the $x$-axis $(y=0)$. In the present shock-rest-frame model, a shock wave is excited by the relaxation of the two plasmas with different quantities. Figure 1 shows that the shock front appears and disappears at a timescale of the downstream ion gyro-period, which corresponds to the cyclic reformation of high Mach number perpendicular shocks. Since the initial state is given by the shock jump conditions for a two-fluid plasma consisting of electrons and ions, the excited shock is "almost" at rest in the simulation domain. The shock front shifts upstream at a very slow velocity of $\sim 0.06 u_{x 1}$, because dynamics of reflected ions is not taken into account in the two-fluid approximation. We have also performed a 1D simulation with the same parameter as the $2 \mathrm{D}$ simulation and confirmed that the period of cyclic reformation in both simulations is the same.

Figure 2 shows the shock-normal electric field $E_{x}$ as a function of position $x$ and time $t$ on the $x$-axis $(y=0)$. There is a good correlation between Figures 2 and 1, implying that the behavior of a shock-normal electric field is controlled by the shock reformation process. Figure 2 shows a bipolar signature of shock-normal electric field turning from negative to positive in the spatial scale of ion gyroradius from $x / \rho_{i}=-0.2$ to 0.2 . The ion-scale electric field corresponds to the shock potential which reflects ions upstream. In the shock foot region there exist sinusoidal wave structures, which are excited by the current-driven instability due to reflected ions. These 


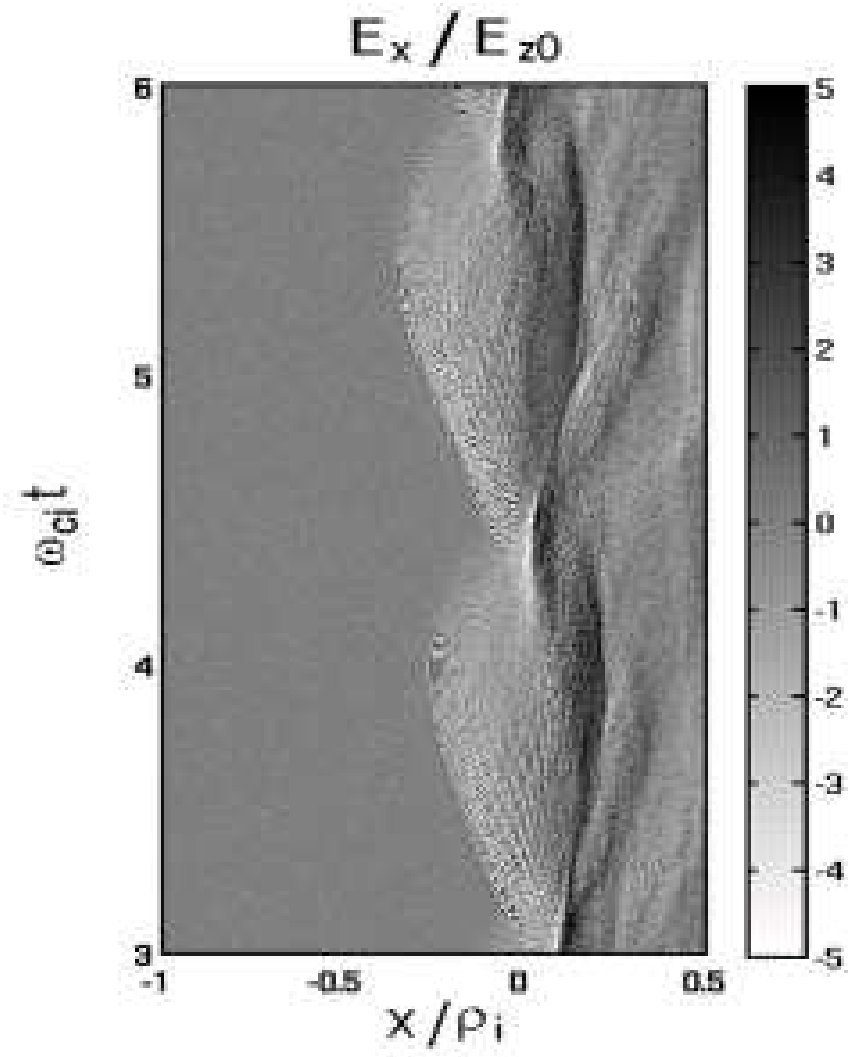

FIG. 2.- Shock-normal electric field $E_{x}$ as a function of position $x$ and time $t$ on the $x$-axis $(y=0)$. The position and time are normalized by $\rho_{i} \equiv u_{x 1} / \omega_{c i 1}$ and $1 / \omega_{\text {cil }}$, respectively. The magnitude is normalized by the motional electric field $E_{z 0}$.

features are also seen in the 1D simulation (not shown here). However, there are two differences between the 1D and 2D results. The first is that the amplitude of electric field in the $2 \mathrm{D}$ simulation is much smaller than that in the $1 \mathrm{D}$ simulation. The second is that the $1 \mathrm{D}$ simulation shows more complex wave structures with several large-amplitude pulses. The typical amplitude of coherent solitary structures in the previous $1 \mathrm{D}$ simulations was $E_{x} / E_{z 0} \sim 20$ and the maximum amplitude exceeded $E_{x} / E_{z 0} \sim 40$ (Shimada \& Hoshino 2000; Hoshino \& Shimada 2002). In the present 2D simulation, on the other hand, the typical amplitude of a shock-normal electric field is $E_{x} / E_{z 0} \sim 5$ and the maximum amplitude is $E_{x} / E_{z 0} \sim 10$. This result suggests that the saturation level of the current-driven instability in the present $2 \mathrm{D}$ simulation is much lower than that in the previous 1D simulation.

Figure 3 shows snapshots of position-velocity phase-space distribution functions of electrons and ions at $\omega_{c i 1} t=4.91$ and the corresponding spatial profiles of $E_{x}$ and $B_{x}$ components. At this time, there is a strong ion beam reflected upstream. Note that the $x-v_{x}$ phase-space distribution functions are reduced by integrating over $y, v_{y}$, and $v_{z}$, and the $x-v_{y}$ phasespace distribution function is reduced by integrating over $y$, $v_{x}$, and $v_{z}$.

Strong heating of electrons is found from the phase-space plots in Fig. 3 in the shock-normal direction in the shock foot region $\left(x / \rho_{i}=-0.2-0\right)$ and at the shock overshoot $\left(x / \rho_{i}=0.1-\right.$ $0.3)$. The shock-normal electric field $E_{x}$ is strongly enhanced by the current-driven instability due to reflected ions in the shock foot region. An electron phase-space vortex is also identified at the leading edge of reflected ions $\left(x / \rho_{i} \sim-0.2\right)$.

\section{Electron}
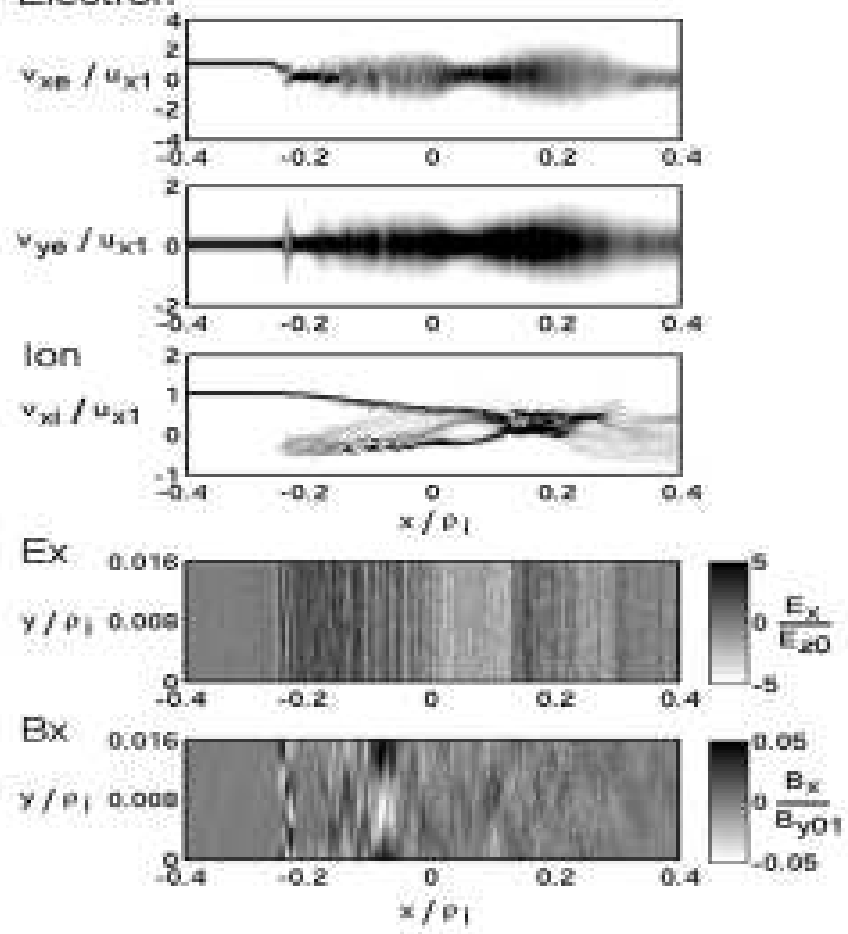

FIG. 3.- Position-velocity phase-space distribution functions of electrons and ions in $x-v_{x e}, x-v_{y e}$, and $x-v_{x i}$ phase spaces. at $\omega_{c i 1} t=4.91$, and the corresponding spatial profiles of $E_{x}$ and $B_{x}$ components. Note that the $x-v_{x}$ phase-space distribution functions are reduced by integrating over $y, v_{y}$, and $v_{z}$, and the $x-v_{y}$ phase-space distribution function is reduced by integrating over $y, v_{x}$, and $v_{z}$.

These results are consistent with the previous 1D simulations.

The beam-plasma interaction in the shock-normal direction results in strong temperature anisotropy $T_{e \perp} / T_{e \|}>10$. We found enhancement of the shock-normal magnetic component $B_{x}$ especially in the electron phase-space vortex and in the shock foot region, which corresponds to electromagnetic whistler mode waves due to electron temperature anisotropy. However, the amplitude of the whistler mode is not so strong that the structure of the transverse magnetic field $B_{y}$ is not modified, keeping an almost 1D structure.

In the electron $x-v_{y}$ phase-space plot, the strong heating of electrons is found in the electron phase-space vortex, in the shock foot region, and at the shock overshoot. These electrons are thermalized along the ambient magnetic field by the whistler mode wave. The electron temperature anisotropy, ensuing excitation of electromagnetic whistler mode waves, and electron heating along the ambient magnetic field would be common features in perpendicular shocks. However, these were not seen in the previous 1D simulation.

Figure 4 shows energy distribution functions of electrons in the downstream region for $1 \mathrm{D}$ and $2 \mathrm{D}$ simulations. The solid line shows the $2 \mathrm{D}$ simulation result, and the dashed line shows the $1 \mathrm{D}$ simulation result. It is known that there appear nonthermal electrons above the upstream bulk energy of ions in 1D simulations (Shimada \& Hoshino 2000; Hoshino \& Shimada 2002). On the other hand, we found less nonthermal electrons in the present $2 \mathrm{D}$ simulation.

The previous 1D studies reported electron surfing acceleration with coherent electrostatic solitary structures in the transition region (Shimada \& Hoshino 2000; Hoshino \& Shimada 2002; Schmitz et al. 2002a. b; Umeda \& Yamazaki 2006). In 


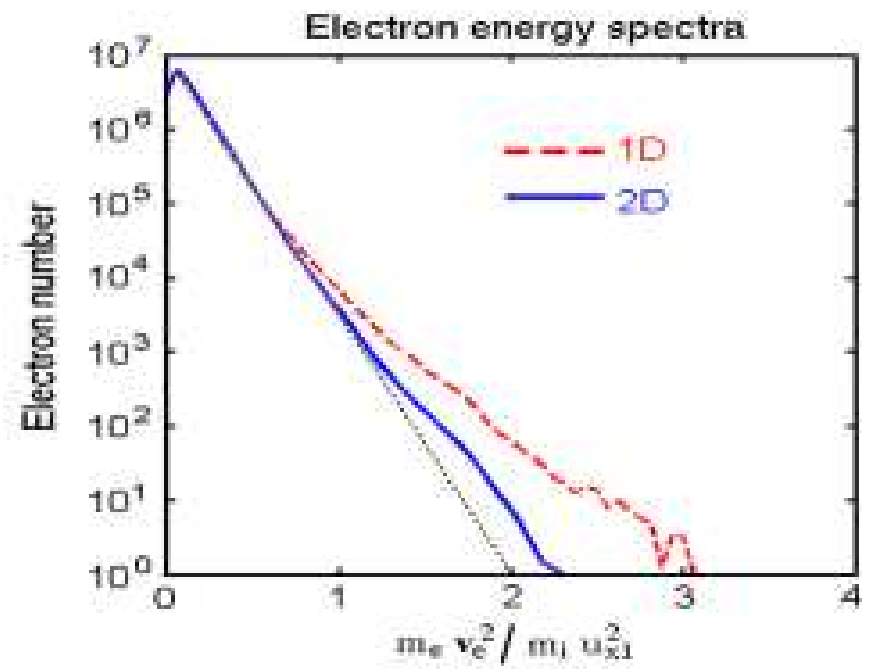

FIG. 4.- Energy distribution functions of electrons in the downstream region for $1 \mathrm{D}$ and $2 \mathrm{D}$ simulations. The solid line shows the $2 \mathrm{D}$ simulation result and the dashed line shows the $1 \mathrm{D}$ simulation result. The dotted line indicates Maxwellian distribution. The energy is normalized by the upstream bulk energy of ions.

the present $2 \mathrm{D}$ simulation, however, we could not confirm the existence of solitary structures in the shock foot region. It is well known that coherent electrostatic solitary structures generated by nonlinear beam-plasma interactions need a strong guiding magnetic field to maintain an electrostatic equilibrium state, the so-called BGK mode (Bernstein et al. 1957). In the present simulation model, electrostatic fields appear in the $x$-direction, while a weak ambient magnetic field is taken in the $y$-direction. Since there is no guiding magnetic field in the $x$-direction, electrons can freely move along the ambient magnetic field, by which electrostatic waves diffuse. As a result, the saturation level of electrostatic waves becomes much lower than that with a strong guiding magnetic field (e.g., Morse \& Nielson 1969; Muschietti et al., 2000; Umeda et al. 2006), and the excited electrostatic waves do not develop into coherent solitary structures.

In addition, waves can also propagate along the ambient magnetic field in the present 2D model. Electromagnetic whistler mode waves are excited by temperature anisotropy, which is caused by electron heating in the shock-normal direction due to current-driven instability. The excited whistler mode waves propagate along the magnetic field and thermalize electrons in the direction parallel to the ambient magnetic field. As a result, we found strong heating of electrons along the magnetic field instead of electron surfing acceleration across the magnetic field. We did not find any further acceleration by the whistler mode waves.

\section{SUMMARY AND DISCUSSION}

We developed a 2D shock-rest-frame model for full particle simulations. Then a 2D simulation of a low-beta and high Mach number perpendicular shock was performed, which shows that electrons are thermalized in the direction parallel to the ambient magnetic field in contrast to the previous $1 \mathrm{D}$ simulations. The heating of electrons along the ambient magnetic field is due to electromagnetic whistler mode waves excited by temperature anisotropy. In addition, the currentdriven instability due to reflected ions does not lead to formation of coherent electrostatic solitary structures because of the free motion of electrons along the ambient magnetic field. As a result of these consequences, the generation of nonthermal electrons via electron surfing acceleration due to electrostatic solitary structures is suppressed in the $2 \mathrm{D}$ system. This process is independent of shock parameters such as $\omega_{p e} / \omega_{c e}$, $\beta$, and $M_{A}$. Even with the realistic parameters, there would be less surfing acceleration in purely perpendicular shocks, because there is no guiding magnetic field to stabilize coherent electrostatic structures. However, the surfing acceleration might be effective in an oblique shock in which a guiding magnetic field could allow the formation of large-amplitude electrostatic structures.

It is noted that Ohira \& Takahara (2007) have also shown that electron surfing acceleration becomes absent by a simplified 2D simulation with different shock geometry. That is, their simulation domain has been taken in a plane perpendicular to the ambient magnetic field (which corresponds to the $x-z$ plane in the present model). Thus, electrostatic solitary structures generated by the current-driven instability lose coherency by a quite different mechanism. However, both 2D simulations with different axes of coordinate have indicated that the electron surfing acceleration is suppressed more than in $1 \mathrm{D}$ simulations. Hence, another unknown injection mechanism for electron acceleration might be necessary.

The computer simulation was carried out on Fujitsu HPC2500 at ITC in Nagoya Univ. and NEC SX-7 at YITP at Kyoto Univ. as a collaborative computational research project at STEL at Nagoya Univ. and YITP at Kyoto Univ. This work was supported in part by Grant-in-aid for Creative Scientific Research 17GS0208 (T. U.) and Grant-in-aid from the Ministry of Education, Culture, Sports, Science, and Technology (MEXT) of Japan, No. 18740153, No. 19047004 (R. Y.).

\section{REFERENCES}

Amano, T., \& Hoshino, M. 2007, ApJ, 661, 190

Bamba, A., Yamazaki, R., Ueno, M., and Koyama, K. 2003, ApJ, 589, 827

Bamba, A., et al. 2005, ApJ, 621, 793

Bernstein, I. B., Greene, J. M., \& Kruskal, M. D. 1957, Phys. Rev., 108, 546

Blandford, R. D. \& Eichler, D. 1987, Phys. Rep. 154, 1

Drury, L. O'C. 1983, Rep. Prog. Phys. 46, 973

Hoshino, M. \& Shimada, N. 2002, ApJ, 572, 880

Hudson, P. D. 1970, Planet. Space Sci., 18, 1611

Koyama, K. et al. 1995, Nature, 378, 255

Lee, R. E., Chapman, S. C., \& Dendy, R. O. 2004, ApJ, 604, 187

Leroy et al. 1981, Geophys. Res. Lett., 8, 1269

Leroy, M. M. et al. 1982, J. Geophys. Res., 87, 5081

McClements, K. G. et al. 2001, Phys. Rev. Lett., 87, 255002

Morse, R. L., \& Nielson, C. W. 1969, Phys. Rev. Lett., 23, 1087
Muschietti, L. et al. 2000, Phys. Rev. Lett., 85, 94

Muschietti, L., Lembege, B. 2006, Adv. Space Res. 37, 483

Ohira, Y., \& Takahara, F. 2007, ApJ, 661, L171

Schmitz, H., Chapman, S. C., \& Dendy, R. O. 2002a, ApJ, 570, 637

Schmitz, H., Chapman, S. C., \& Dendy, R. O. 2002b, ApJ, 579, 327

Shimada N., \& Hoshino, M. 2000, ApJ, 543, L67

Umeda, T. 2004, Ph.D. Thesis, Kyoto University

Umeda, T. et al. 2001, Comput. Phys. Commun., 137, 286

Umeda, T. et al. 2003, Comput. Phys. Commun., 156, 73

Umeda, T. et al. 2006, J. Geophys. Res., 111, A10206.

Umeda, T. \& Yamazaki, R. 2006, Earth Planets Space, 58, e41 (arXiv: physics/0607220 